ROCZNIKI HUMANISTYCZNE

Tom LXVIII, zeszyt 5 - 2020

DOI: http://dx.doi.org/10.18290/rh20685-7

GABRIELE VON GLASENAPP

\title{
„DER DOKTOR BLEIBT!“‘: JANUSZ KORCZAK ALS LITERARISCHE FIGUR
}

\begin{abstract}
A b strakt. Der Beitrag untersucht die literarische Repräsentation von Janusz Korczak in der europäischen Kinder- und Jugendliteratur der vergangenen vierzig Jahre. Dabei wird zugleich auf die Besonderheiten von Kinder- und Jugendliteratur als ein genuin plurimediales Erinnerungsmedium eingegangen, in dem sowohl auf Text- und auf Bildebene an die Shoah erinnert wird. In besonderer Weise perspektiviert wird außerdem der transnationale Charakter von Kinder- und Jugendliteratur, der sich auch in den zahlreichen Erzählungen über Janusz Korczak manifestiert. Durch den Akt von wechselseitigen Übersetzungen werden die Texte zu einem Bestandteil mehrerer Literaturen, so dass hier durchaus von Weltliteratur in dem Sinne gesprochen werden kann, dass sie sich nicht nur in ein nationales oder bi-nationales, sondern in ein globales kulturelles Gedächtnis einschreiben.
\end{abstract}

Schlüsselwörter: Janusz Korczak; Kinder- und Jugendliteratur; Shoah; kulturelles Gedächtnis.

\section{EINLEITENDE BEMERKUNGEN ${ }^{1}$}

$\mathrm{Zu}$ der Vielzahl an unauflösbaren Paradoxien der Shoah zählt die Tatsache, dass die in sie involvierten, von ihr betroffenen Nationen dieses von Deutschen verursachten Genozides nunmehr, bald achtzig Jahre danach, gemeinsam dieser Ereignisse gedenken und auch gemeinsam deren vielfältige mediale Repräsentation erforschen. Dabei befinden sich die Forscher an einem Zeitpunkt, an dem nur noch wenige Zeitzeugen leben, d.h. sich das kommunikative unaufhaltsam in ein kulturelles Gedächtnis wandelt, was nichts anderes bedeutet, dass Medien bald endgültig den Platz von Zeitzeugen eingenommen haben werden. Nur sie

Prof. Dr. GABRIELE von GlaSenAPP - Universität zu Köln, Philosophische Fakultät, ALEKI Arbeitsstelle für Kinder- und Jugendmedienforschung; Korrespondenzadresse: Bernhard-Feilchenfeld-Str. 11, 50969 Köln; E-Mail: g.glasenapp@uni-koeln.de. ORCID: https://orcid.org/00000002-3794-6700.

${ }^{1}$ Der Beitrag ist eine erweiterte und neu perspektivierte Fassung meines Aufsatzes über kinder- und jugendliterarische Repräsentationen von Janusz Korczak (Glasenapp). 
(einschließlich aller zur Verfügung stehenden Quellen) werden noch davon berichten können, was sich zugetragen hat. Auch das ist eine Gemeinsamkeit aller Nationen, in deren kulturellem Gedächtnis die Shoah einen Platz hat, wie auch die Tatsache, dass diese Nationen mehrheitlich auf dieselben Erinnerungsmedien zurückgreifen, wenn es gilt, die Shoah auch in das kulturelle Gedächtnis der nachfolgenden Generationen zu implementieren.

Dass auch und gerade literarische Texte in allen denkbaren Formaten (u.a. Autobiographien, Biographien, Romane, Erzählungen, Novellen) von Beginn an bis in die Gegenwart zu den wichtigsten Erinnerungsmedien zählen, muss nicht gesondert betont werden; schon vor geraumer Zeit hat Astrid Erll nicht nur darauf hingewiesen, in welchem Ausmaß „Texte aller Gattungen und Genres [...] als Medien des kollektiven Gedächtnisses“ fungieren können (249), sondern auch darauf, dass jegliche „Erinnerungen erst durch mediale Repräsentation und Distribution zu kollektiver Relevanz gelangen“ (251).

Spricht man von erzählender Literatur als Erinnerungsmedium, dann wird jedoch bis heute von der Forschung allzu oft ausgeblendet, dass zu dieser erzählenden wie Sachliteratur auch kinder- und jugendliterarische Texte zu zählen sind. Darunter gefasst werden üblicherweise alle Texte, die entweder explizit für Heranwachsende verfasst werden bzw. an sie adressiert sind, zum anderen jene Werke, die Erwachsene Kindern und Jugendlichen zur Lektüre empfehlen, was natürlich auch Werke aus der Erwachsenenliteratur sein können. Zuletzt wird mit Kinder- und Jugendliteratur ein Korpus an Texten bezeichnet, die von Kindern bzw. Jugendlichen im außerschulischen Kontexten, also freiwillig konsumiert werden, eine Konstellation, die eine spätere schulische Lektüre jedoch keineswegs ausschließt (Ewers 14-22).

Analog zur allgemeinen, also Erwachsenenliteratur, ist auch Kinder- und Jugendliteratur von ihrer Entstehung an eine erinnerungskulturelle Funktion eingeschrieben - so existieren bereits im 18. Jahrhundert kinderliterarische Texte, die von als herausragend und daher als ,erinnerungswürdig' erachteten Ereignissen oder Persönlichkeiten erzählen (Brüggemann und Ewers 951-1216), und sie auf diese Weise erinnern. Im Verlauf des 19. Jahrhunderts werden auch in der Kinder- und Jugendliteratur neben Biographien und Geschichtsdarstellungen historische Romane und Erzählungen zu einer der wichtigsten erinnerungskulturellen Gattungen (Wilkending 537-616). In den Blick der Forschung ist diese erinnerungskulturelle Funktion von Kinder- und Jugendliteratur allerdings erst sehr viel später, d.h. in den 1990er Jahren geraten (Glasenapp und Wilkending) ${ }^{2}$.

\footnotetext{
${ }^{2}$ Die Gesellschaft für Kinder- und Jugendliteraturforschung hat sich auf ihrer 32. Jahrestagung im Mai 2019 genau dieser Thematik angenommen: „Kulturelles Gedächtnis reloaded.
} 
Erinnert wird innerhalb der deutschsprachigen Kinder- und Jugendliteratur auch an den Genozid an den europäischen Juden sowie an anderen Minderheiten; seit Anfang der 1960er Jahre existiert ein kaum noch zu überschauendes Korpus an erzählenden wie an Sachtexten über die Shoah (vgl. u.a. Glasenapp und Ewers, Oeste und Preußer, Zimmermann) - und zwar für alle Altersstufen. Allerdings ist analog zur allgemeinen Literatur auch die Kinder- und Jugendliteratur der letzten Jahrzehnte von dem Phänomen des generationalen Wandels betroffen; mittlerweile schreiben auch hier mehrheitlich Autorinnen und Autoren der zweiten und dritten Post-Shoah-Generation und zwar für Rezipienten, die alle nach der Jahrtausendwende geboren sind, so dass hier bereits von einer vierten und fünften Generation gesprochen werden muss.

In ihren inhaltlichen Schwerpunktsetzungen orientiert sich die Kinderund Jugendliteratur bei ihren Erzählungen über die Shoah mehrheitlich an der allgemeinen, also der Erwachsenenliteratur, dennoch gibt es gerade hier auch signifikante Unterschiede, und diesen Unterschieden, ja, Alleinstellungsmerkmalen sollen die folgenden Ausführungen gewidmet sein und zwar: auf der Gegenstandsebene, hinsichtlich der Plurimedialität von Kinder- und Jugendliteratur sowie im Hinblick auf ihre Transnationalität.

\section{DIE GEGENSTANDSEBENE: JANUSZ KORCZAK ALS HISTORISCHE PERSÖNLICHKEIT UND LITERARISCHE FIGUR}

Begonnen werden soll mit der Gegenstandsebene: In einer Abhandlung über die erinnerungskulturellen Aspekte der Shoah muss nicht näher erläutert werden, wer Janusz Korczak (1878/79-1942) war und aus welchem Grund er mittlerweile eine der wichtigsten und auch bekanntesten Erinnerungsfiguren der jüngeren polnischen wie europäischen Geschichte ist. Nach Janusz Korczak, zu Lebzeiten ein ebenso bedeutender wie bekannter und populärer Kinderarzt, Pädagoge und Kinderbuchautor, sind Schulen und Straßen benannt, ihm wurden zahlreiche Biographien gewidmet, Denkmäler errichtet, es gibt Periodika und Literaturpreise, die seinen Namen tragen, Filme und Theaterstücke, die die Geschichte seines Lebens behandeln, seine wissenschaftlichen, publizistischen wie kinderliterarischen Werke werden bis heute aufgelegt, und - seit langem schon sind sowohl sein Leben wie seine

(Re-)Inszenierungen von Erinnerung in Kinder- und Jugendmedien“ (http://www.gkjf.de/wpcontent/uploads/2019/02/Programm-GKJF-2019.pdf; 3.8.2019). Die Publikation des Tagungsbandes ist für 2020 vorgesehen. 
Forschungen Gegenstand unterschiedlicher wissenschaftlicher Disziplinen, darunter vor allem der allgemeinen wie der Heil- und Sonderpädagogik, der Kinderpsychologie wie der Kindheitsforschung - und zwar keineswegs nur in Polen und Deutschland, sondern nahezu weltweit. Es wird also in sehr unterschiedlichen Kontexten und Medien über den Pädagogen Janusz Korczak, erzählt', wenngleich - erstaunlich genug - bislang kaum Forschungen zu seiner medialen Repräsentation existieren. Eine der wenigen Ausnahmen betrifft den Sammelband Korczakian Reminiscences (Chłosta-Sikorska, StępniewskaGębik, Trojański) dessen erster Teil zwar überschrieben ist mit „Korczak Portrayed: Representations in Literature and Visual Art" (13-63); allerdings legen die darunter versammelten Beiträge ihren Fokus vornehmlich auf die Darstellung von Korczak in polnischen Biographien, in Filmen sowie in den bildenden Künsten, während die erzählenden, literarischen Zeugnisse ausgespart bleiben.

Wenn über Janusz Korczak im weitesten Sinne ,erzählt' und damit an ihn erinnert wird, an den Mann, der von den Deutschen gezwungen wurde, im Oktober 1940 mit den Kindern des von ihm geleiteten jüdischen Waisenhauses in das Warschauer Ghetto umzusiedeln, bevor er im August 1942 zusammen mit den Kindern sowie seinen Mitarbeiterinnen und Mitarbeitern nach Treblinka deportiert und ermordet wurde, so betrifft das vor allem den Moment seiner Deportation. Mehrere Zeitgenossen, von denen jedoch nur einer die Shoah überlebt hat, haben Zeugnis abgelegt vom Marsch der Kinder mit Janusz Korczak vom Waisenhaus zu den Güterzügen. Es ist vor allem diese Szene, wiewohl sie nicht in einem Bildmedium festgehalten worden ist, die den Arzt, Pädagogen und Kinderbuchautor Janusz Korczak bereits in den 1950er Jahren neben Anne Frank zu einer der zentralen Projektionsfiguren, ja, zu einer Ikone der Opfer der Shoah werden ließ. Kaum ein Ereignis der Shoah hat eine vergleichbare Wirkung entfaltet und keines auch mehr Zuneigung erfahren als die Erzählung über den Arzt, der , seine' Kinder freiwillig in den Tod begleitet, Kinder, die ihm - eine Wissenshierarchie, die für den ikonographischen Charakter dieser Szene von zentraler Bedeutung ist - ahnungslos und voller Vertrauen folgen. Es ist diese Szene, die, zumindest in der Wahrnehmung der Überlebenden und nachfolgenden Generationen, zudem gleichsam als Inkarnation, als Matrix für den Genozid an den europäischen Juden erscheint, und eine Szene, der zugleich alle Züge eines sogenannten Masternarrativs eingeschrieben sind. Es ist dieser letzte, eigentlich vorletzte Gang von Korczak und ,seinen“ Kindern, der allen erinnerungskulturellen Überlieferungsformen in letzter Konsequenz zugrunde liegt: 
„Man kann nichts von Korczak lesen, nichts über ihn hören, ohne daran zu denken, wie sein Weg endete, ohne den Zug der Kinder über die Straßen des Warschauer Ghettos, zur Verladerampe, zur ,Endlösung“ vor sich zu sehen; jenen Zug jüdischer Waisenkinder, denen ihr Erzieher, ihr ,Herr Doktor', der Arzt, Schriftsteller, Pädagoge Dr. Henryk Goldszmit, voranschritt, ihnen auch in diesem Augenblick Zuverlässigkeit, Vertrauen und Menschsein gebend. ... Die Legende erzählt - wenn das auch durch Augenzeugen nicht belegt ist, also wohl nie mehr authentisch zu machen sein wird, trotzdem aber doch stimmend, historisch passend, in diesem Sinne also authentisch -, daß dem Zug der jüdischen Waisenkinder am glühendheißen Augusttag 1942 zur Verladerampe, in die Waggons, die sie nach Treblinka brachten, diese grüne Fahne vorangetragen worden sei“ (Schulze 446 f.).

So Günter Schulze 1978 in seinem Nachwort zu Janusz Korczaks bekanntestem Kinderroman König Macius der Erste, so auch der Tenor in allen anderen Veröffentlichungen, die sich mit Korczaks pädagogischen Konzepten und mit seiner Biographie beschäftigen. Bereits an dieser Stelle wird deutlich, dass es nicht ausschließlich den Ereignissen selbst geschuldet ist, dass Janusz Korczak mittlerweile zum festen Bestandteil des kulturellen Gedächtnis zählt, sondern der, Übersetzung' der Geschichte in fiktionale Narration, in den Akt des Erzählens und der mit diesem Akt untrennbar verbundenen performativen Geste - denn Geschichte ist immer erzählte Geschichte, die sich zwangsläufig imagologisch organisiert und diskursiven Prägungen unterliegt (vgl. Ahrens 47) - das gilt auch und gerade für , die Geschichte' über Janusz Korczak und seine Kinder.

Wenn sich diese Ausführungen nun mit Erzählungen über Janusz Korczak beschäftigen, dann jedoch aus einer Perspektive, die bislang nur ansatzweise im Fokus der Forschung gestanden hat - nämlich mit kinder- und jugendliterarischen Repräsentationen von Janusz Korczak, d.h. inwieweit sie dazu beitragen, die Figur Korczak und hier vor allem die Umstände seines Todes, in das kulturelle Gedächtnis der jetzigen wie der späteren Generationen einzuschreiben.

Auch bei einem nur kursorischen Überblick über die literarischen, fiktionalen Texte, die die Geschichte von Janusz Korczak und ,seinen Kindern' verhandeln, zeigt sich, dass es von den Anfängen bis in die unmittelbare Gegenwart nahezu ausschließlich kinder- bzw. jugendliterarische Werke sind, in denen über Korczak erzählt wird. Damit besitzt die Kinder- und Jugendliteratur, was diesen spezifischen Teil der Erinnerungskultur betrifft, gegenüber der allgemeinen Literatur eindeutig ein Alleinstellungsmerkmal. Dane- 
ben gibt es auch kinder- und jugendliterarische Sachtexte, die das Leben und Sterben von Korczak behandeln, am bekanntesten ist hier zweifellos die mehrfach aufgelegte und übersetzte Biographie von Monika Pelz (1985), des Weiteren an Heranwachsende adressierte Abhandlungen über den Holocaust oder über bedeutende Persönlichkeiten der Geschichte ${ }^{3}$, in denen Korczak ebenfalls genannt wird - aus Platzgründen soll sich in dieser Abhandlung jedoch ausschließlich auf die fiktionalen Formate beschränkt werden.

Die Erzählungen über Janusz Korczak nehmen ihren Anfang unmittelbar nach seiner Ermordung - zunächst in Form von Zeitzeugenberichten, die alle den Moment von Korczaks Deportation, d.h. den Marsch von ihm, seinen Mitarbeitern und den Kindern zum ,Umschlagplatz' sowie die Situation dort ins Zentrum stellen, und zum Teil sehr zeitnah, d.h. noch in den 1940er Jahren, veröffentlicht werden, zum Teil jedoch auch erst in den folgenden Jahrzehnten ${ }^{4}$. Ein erstes literarisches Zeugnis (nicht an Heranwachsende adressiert) bildet der 1946 in Palästina veröffentlichte Roman Ha doktor nish'ar. Roman biografi al Yanush Korczak (Der Doktor bleibt. Biografischer Roman über Janusz Korczak) der polnischen Publizistin und Übersetzerin Pola Apenszlak (d.i. Paulina Appenszlakowa). Appenszlakowa war die Frau des bekannten Publizisten Jakób Appenszlak (1894-1950), seit 1923 Herausgeber der polnisch-jüdischen Tageszeitung Nasz Przeglad (Unsere Rundschau), deren Kinderbeilage Maty Przeglad (Kleine Rundschau) von Korczak betreut wurde (Steffen 78). Es ist anzunehmen, dass der Impuls, einen Roman über das Leben und Sterben von Korczak zu verfassen, auf dieser langjährigen

\footnotetext{
${ }^{3}$ Vgl. u.a. Bruchfeld/Levine 67; Lawton 30; Lewin, "Der Wind trägt die Worte" 395-399; Nürnberger 171-192.

${ }^{4}$ Darunter der jiddische Schriftsteller Jehoshua Perle (1888-1943), der kurz darauf selbst in Auschwitz ermordet wurde, in seiner, im Oktober 1942 beendeten Chronik Churbn Warsze (Die Zerstörung Warschaus) (Berenstein 313 f.); Nahum Remba (1910-1943), ein Mitglied des Judenrats, der letzte, der mit Korczak noch auf dem Umschlagplatz gesprochen hat (vgl. Eisenbach 606; eine deutsche Übersetzung findet sich in Sakowska 65), sowie der Pianist Władysław Szpilman (1911-2000). Sowohl die Chronik von Perle wie auch die Erinnerungen von Remba sind Teil des 1946 in den Trümmern des Warschauer Ghettos geborgenen (Geheimen) Ringelblum-Archivs und mittlerweile vielfach ediert worden. Am frühesten publiziert (1946) wurden die Erinnerungen an Korczaks Deportation von Władysław Szpilman, zugleich dem einzigen Überlebenden der hier Genannten (101-103); eine Neuausgabe u.d.T. Das wunderbare Überleben. Warschauer Erinnerungen 1939-1945, die zugleich als Grundlage für den Film Der Pianist von Roman Polański diente, wurde 1998 in deutscher Sprache von Szpilmans Sohn Andrzej Szpilman herausgegeben und mittlerweile in zahlreiche Sprachen übersetzt. Zum Bericht über die Deportation Korczaks vgl. 93 f. $\mathrm{Zu}$ den unterschiedlichen Versionen und deren z.T. „legendenhaften Charakter" vgl. Lewin, "So war es wirklich"132-136.
} 
persönlichen Bekanntschaft zwischen dem Ehepaar Appenszlak und Korczak beruhte. Anders als dieser befanden sich sowohl Jakób als auch Paulina bei Beginn des Krieges nicht in Warschau; Jakób konnte Anfang September 1939 nicht mehr nach Polen zurückkehren und floh in die USA, Paulina entkam in letzter Minute mit ihrem Sohn nach Palästina (Steffen 371). Die hebräische Version von Appenszlakowas Roman beruhte auf einem nicht veröffentlichten Manuskript in polnischer Sprache, das von Chaim Shalom BenAvram ins Hebräische übersetzt wurde. Obwohl der Roman in Israel 1953 sogar eine zweite Auflage erlebte, 1961 von Diana Blumenfeld (1903-1961), einer bekannten polnischen Schauspielerin und Sängerin, die sowohl das Warschauer Ghetto als auch das Konzentrationslager Majdanek überlebt hatte, ins Jiddische übersetzt wurde ${ }^{5}$ und kurz darauf (1963), ebenfalls in Buenos Aires, auch eine spanische Übersetzung erschien ${ }^{6}$, blieb die Rezeption zwangsläufig auf einen kleinen Adressatenkreis beschränkt.

Sehr viel mehr Resonanz war dem Theaterstück Korczak und die Kinder von Erwin Sylvanus (1917-1985) beschieden, das im November 1957 seine Uraufführung in Deutschland erlebte, bald zu den meistgespielten deutschen Theaterstücken der Nachkriegszeit zählte und in zahlreiche Sprachen übersetzt wurde (vgl. Gödden 160). Erstmals wurde auf diese Weise die Aufmerksamkeit der deutschen Öffentlichkeit auf den bis dahin in Deutschland vollkommen unbekannten Pädagogen und sein Schicksal gelenkt - und damit auch auf die unmittelbare deutsche Vergangenheit - und zwar etliche Jahre vor den Eichmann- und Auschwitzprozessen und auch vor den Theaterstücken von Rolf Hochhuth und Peter Weiss.

Knapp zwanzig Jahre später war es der von dem deutschen Filmproduzent Artur Brauner (1918-2019) initiierte deutsch-israelische Spielfilm Sie sind frei, Dr. Korczak (1974), der entscheidend dazu beitrug, Janusz Korczak als Erinnerungsfigur im kulturellen Gedächtnis der deutschen Nachkriegsgenerationen zu verankern.

Dass sich die Kinder- und Jugendliteratur in den 1970er Jahren der Persönlichkeit und dem Schicksal Korczaks zuwendete, ist aber nicht nur diesen

\footnotetext{
${ }^{5}$ Die Übersetzung erschien unter dem leicht veränderten Titel Januš Koršak biografišer roman und hob im Untertitel explizit hervor, dass es sich um eine „oitorizirte iberzetzung fun poiliše original“" handelte, d.h. offensichtlich hatte Blumenfeld von Appenszlakowa das polnische Originalmanuskript erhalten.

${ }^{6}$ Auch die spanische Übersetzung erschien unter einem neuen Titel Una luz en las tinieblas. Vida y pasión de Janusz Korczak. Novela (Ein Licht in der Finsternis. Leben und Leidenschaft von Janusz Korczak. Roman). Die Vorlage für die beiden Übersetzer Luis Kardúner und Abraham Rosenblum bildete nun die jiddische Übersetzung von Diana Blumenfeld.
} 
ersten medialen Vergegenwärtigungsversuchen in Literatur und Film geschuldet, sondern auch einer zunehmenden gesellschaftlichen Auseinandersetzung mit der Shoah, die in Deutschland schließlich in die postume Verleihung des Friedenspreises des Deutschen Buchhandels an Janusz Korczak im Jahre 1972 mündete (Scheideler 314) und daran anschließend in eine verstärkte Publikation von Korczaks Werken. In Polen wiederum war es der hundertjährige Geburtstag Korczaks, der seinerzeit auf das Jahr 1979 festgelegt wurde, denn in diesem Jahr erschien der Jugendroman Czarodziej (Der Zauberer) der bekannten Autorin Wanda Żółkiewska (1912-1989), dessen Titel bereits unübersehbar auf Korczak verweist, spielt er doch auf seinen 1935 erschienenen Kinderroman Kajtuś Czarodziej (Kaitus der Zauberer) an. Eine deutsche Übersetzung von Żółkiewskas Roman erschien erst zwei Jahre später (1981) in der DDR unter dem deutlich weniger aussagekräftigen und seine Thematik eigentlich verbergenden Titel Eine Chance für Heniek. Damit markiert Żółkiewskas Roman über einen jüdischen Jugendlichen (Heniek), der Zuflucht im Waisenhaus von Janusz Korczak findet, den Beginn kinder- bzw. jugendliterarischen Erzählens über Janusz Korczak, und auch auf inhaltlicher Ebene finden sich Schwerpunktsetzungen, die in den folgenden Jahren und Jahrzehnten von kinder- und jugendliterarischen Erzählungen fortgeschrieben werden: Mehrheitlich werden die Ereignisse nicht aus der Perspektive der Erwachsenen, sondern aus jener der heranwachsenden, der kindlichen Akteure übermittelt. Weiterhin begründet Żółkiewskas Roman eine publizistische Tradition, die ebenfalls bis heute anhält: Während Korczaks wissenschaftliche und publizistische Werke ausschließlich an Erwachsene adressiert sind, richten sich die fiktionalen Erzählungen über Korczak nahe$\mathrm{zu}$ ebenso ausschließlich an heranwachsende Leser - und zwar an Kinder ebenso wie an Jugendliche.

Bis heute sind mehr als zwanzig kinder- und jugendliterarische Werke erschienen, in denen die Figur Janusz Korczak mit sehr unterschiedlichen Schwerpunktsetzungen in Erscheinung tritt. Die Bandbreite reich von Erzählungen, in denen die Figur Korczak im Zentrum steht (was reicht vielfach bereits im Titel herausgestellt wird) (vgl. Adler und Ritz, Bernheim, Bogacki, Chmielewska, Cohen-Janka und Quarello, Einat und Kelner, Kang und Hye-yŏng, Laird, Neudeck und Ruegenberg, Spielman und Archambault) bis hin zu Texten, die zwar im Warschauer Ghetto angesiedelt sind, in denen Korczak jedoch lediglich eine Nebenfigur ist oder auch nur erwähnt wird (vgl. Adler und Ritz, Du Bouchet, Gleitzman, Jaromir und Cichowska, Mingarelli, Safier, Shem-Tov, Shepard, Stoffels, Szczygielski, Vandewijer, 
Welsh). Darunter finden sich sowohl jugend- wie kinderliterarische Romane (vgl. Du Bouchet, Gleitzman, Jaromir und Cichowska, Laird, Mingarelli, Safier, Shem-Tov, Shepard, Stoffels, Szczygielski, Vandewijer, Welsh, Żółkiewska), mehrere erzählende sowie Sachbilderbücher bzw. Graphic Novels (vgl. Adler und Ritz, Adler und Farnsworth, Bernheim, Bogacki, Chmielewska, Cohen-Janca und Quarello, Einat, Kang und Hye-yŏng, Neudeck und Rügenberg, Spielman und Archambault), auf die noch einmal gesondert eingegangen werden soll. Eine deutliche Massierung der Werke seit den 2010er Jahren ist unübersehbar, was sicherlich auch in unmittelbarem Zusammenhang mit dem 2012 vom polnischen Parlament ausgerufenen internationalen Janusz-Korczak-Jahr steht (erinnert wurde dabei sowohl an den 70. Jahrestag von Korczaks Ermordung im KZ Treblinka als auch an das 100jährige Jubiläum der Eröffnung von Korczaks erstem Waisenhaus im Oktober 1912).

Bezogen auf die in der zeitgeschichtlichen Kinder- und Jugendliteratur manifest werdenden Erinnerungsstrategien an die Shoah, so kann zunächst festgehalten werden, dass diese intensive, bis heute andauernde und sich beständig wiederholende Beschäftigung mit einer historischen Persönlichkeit, ihrem Leben, ihrem Wirken und vor allem ihrem Sterben ein absolutes Novum darstellt. Keine andere Figur ist innerhalb der erzählenden, fiktionalen Kinder- und Jugendliteratur häufiger behandelt worden als Janusz Korczak nicht einmal Anne Frank, eine Tatsache, die sicherlich mehreren Umständen geschuldet ist: Zum einen der Singularität des Verbrechens - der geplanten und willentlichen Ermordung von über 200 Kindern und ihren Erzieherinnen, ein Verbrechen, das damit gleichsam als Matrix für die Gräuel der Shoah angesehen werden kann - des Weiteren der Besonderheit, dass Korczak nachweislich Angebote hatte, das Ghetto vor der Deportation zu verlassen, worauf in der Mehrheit der Erzählungen auch explizit hingewiesen wird, und nicht zuletzt spielt eine nicht unerhebliche Rolle, dass Korczak bereits vor 1939 nicht nur in Polen aufgrund seiner Publikationen wie seiner medialen Präsenz eine sehr bekannte Persönlichkeit war, dessen Schicksal während der deutschen Besetzung Polens bereits von den Zeitgenossen mit großer Aufmerksamkeit verfolgt wurde.

Betrachtet man nun die mediale Repräsentation, d.h. die Erzählungen über Janusz Korczak in ihrer Gesamtheit, so lassen sich bestimmte inhaltliche Muster erkennen: Auch wenn das von Korczak geleitete Waisenhaus stets als zentraler Schauplatz präsent ist, wird den heranwachsenden Akteuren selbst ein deutlich größerer Aktionsradius zugestanden. Während aller- 
dings die kinderliterarischen Texte mehrheitlich fokussiert sind auf die Topographie des Waisenhauses und den dort von Korczak zur Anwendung gebrachten pädagogischen Maximen, ist die Aufenthaltsdauer jugendlicher Akteure in dieser Heterotopie immer nur von begrenzter Dauer. Und während die kinderliterarischen Texte nahezu ausschließlich vom kindgemäßen Leben im Waisenhaus und dem anschließenden Sterben aller Akteure erzählen, thematisieren die Jugendromane (vgl. Gleitzman, Laird, Safier, Shem-Tow, Shepard, Stoffels, Vandewijer, Żółkiewska) vor allem das Überleben - ihrer jugendlichen Protagonisten. Sie verlassen das Waisenhaus schon vor der Deportation, um später am Ghettoaufstand teilzunehmen oder aus dem Ghetto zu fliehen. Implizit werden auch sie durch den Akt des Aufbruchs (aus dem Waisenhaus) zu Erinnerungsfiguren, denn als Überlebenden fällt ihnen die Aufgabe zu, der Nachwelt von ihrem eigenen Schicksal und damit von Korczak und den Kindern zu erzählen. Dieses Muster scheint bereits in dem Roman von Żółkiewska auf, worauf implizit auch der Titel der deutschen Übersetzung verweist und damit zugleich den Fokus von Korczak (wie in der polnischen Originalversion) auf den jugendlichen Akteur verlagert (Eine Chance für Heniek); es wird in der Folge von allen weiteren Erzählungen immer wieder aufs Neue aufgegriffen und nur geringfügig variiert.

\section{DIE PLURIMEDIALITÄT DER KINDER- UND JUGENDLITERATUR}

Plurimedialität, ein Begriff der der Medien- und Kommunikationsforschung entlehnt wurde (Wilke $303 \mathrm{f}$.), bezeichnet im Kontext der Kinderund Jugendliteraturforschung den Umstand, dass die Kinder- und Jugendliteratur sehr viel häufiger als die allgemeine Literatur Gebrauch von einem zweiten Code macht - jenem der Illustration (Ewers 175 f.). Das betrifft vor allem die Kinderliteratur und auch kinderliterarische Texte über die Shoah, worunter sowohl illustrierte Erzählungen wie auch Bilderbücher und Comics bzw. Graphic Novels gefasst werden. Im Unterschied zur Geschichte der erzählenden Kinder- und Jugendliteratur über die Shoah, die in Deutschland Ende der 1950er Jahre beginnt, erscheinen die ersten Bilderbücher erst knapp dreißig Jahre später, den Anfang macht 1986 das höchst kontrovers diskutierte Werk Rosa Weiss des italienischen Illustrators Roberto Innocenti über ein neunjähriges Mädchen, das Krieg und Deportation (von Kindern) beobachtet, ohne zu verstehen, was vor sich geht und am Ende vermutlich 
selbst ein Opfer des Krieges wird (Thiele).

Mittlerweile zählen Bilderbücher und Comics nicht nur in Deutschland als wichtige kinder- und jugendliterarische Erinnerungsmedien (Dolle-Weinkauff, Frahm); im Falle von Janusz Korczak haben sie maßgeblich dazu beigetragen das ikonische Bild von Korczak und ,seinen' Kindern im kulturellen Gedächtnis nicht nur zu bewahren, sondern auch zu etablieren ${ }^{7}$. Dabei kam den Illustratoren ebenfalls der Umstand von Korczaks starker medialer Präsenz vor Beginn des Krieges entgegen, die sich auch in einer Vielzahl von existierenden Fotos manifestierte. Es sind Bilder wie diese, die mittlerweile tief im kollektiven Gedächtnis verankert sind; auf diese Weise bilden sie gleichsam die bildliche Matrix, die allen Illustrationen - ähnlich wie im Falle von Anne Frank - unterlegt ist. Auf diese Weise tragen sie bis heute entscheidend dazu bei, dass bereits die Illustration allein ausreicht, um dem Betrachter die historische Person Janusz Korczak und sein Schicksal zu vergegenwärtigen.

Mit dazu beigetragen hat auch die Vielzahl der bis heute erschienenen Bilderbücher, die Stationen aus Korczaks Leben, seine gesamte Biographie, seine Interaktion mit den Kindern im Ghetto, die letzten Tage im Waisenhaus oder den Moment der Deportation in Bild und Text erzählen und dabei mehrheitlich Korczak (mit Kindern) bereits auf dem Titelcover zeigen.

Eines der ersten Bilderbücher über Janusz Korczak überhaupt erscheint in Deutschland im Jahr 2000 (Rügenberg/Neudeck) ${ }^{8}$. Erzählt wird auf wenigen Seiten aus der Sicht eines auktorialen Erzählers die Geschichte von Janusz Korczak, dem von ihm geleiteten Waisenhaus und den darin lebenden Kindern vom Beginn des Krieges bis zur Deportation. An der Ermordung der jüdischen Akteure lassen Text und Bilder keinen Zweifel. Bezeichnend ist, dass außer der Figur Korczaks kein anderes Individuum in Erscheinung tritt, alle kindlichen Akteure erscheinen trotz Namensnennung vollkommen entindividualisiert. Auf der Bildebene - und dieser Strategie folgen nahezu alle

\footnotetext{
${ }^{7}$ Bislang existieren zwar zahlreiche Bilderbücher über Janusz Korczak (s. oben), allerdings nur ein Comic, in dem er visuell in Erscheinung tritt (Greinecker-Morocutti 137), auf textueller Ebene wird er nicht genannt. Zu den Gründen vgl. Greinecker-Morocutti 142 f.). Die wenigen Comics bzw. Graphic Novels über das Warschauer Ghetto, darunter vor allem Kubert und Sax, konzentrieren sich vor allem auf die Ereignisse des Warschauer Ghettoaufstandes (vgl. Baskind und Prager).

${ }^{8}$ Lediglich das Bilderbuch Froim - Der Junge aus dem Warschauer Ghetto (Adler und Ritz) ist im amerikanischen Original bereits 1995 erschienen. Es ist symptomatisch für die visuelle Rezeption Korczaks, dass die deutsche Übersetzung erst in deutlichem zeitlichem Abstand (2011) veröffentlicht wurde.
} 
weiteren Bilderbücher - wird immer wieder auf die ikonographisch gezeichnete Figur Korczaks inmitten von Kindern abgehoben. Die Bilderbücher, die im Verlauf der 2010er Jahre erschienen sind, und sich zumindest partiell auch durch ihr hohes ästhetisches Niveau auszeichnen (vgl. v.a. Chmielewska, Jaromir und Cichowska, Cohen-Janca und Quarello), verweisen hingegen, z.T. auch bereits im Titel, nicht nur auf die Existenz individueller kindlicher Akteure, diese werden nun auch explizit als erinnernde Subjekte in Szene gesetzt. Dennoch bleibt auch ihr Fokus in letzter Konsequenz immer auf die Zentralfigur Korczak gerichtet, den auch alle kindlichen Akteure, alle kindlichen Erzähler, immer in den Tod begleiten.

Im Kontext der Erinnerung an die Shoah unterliegen Bilderbücher einem besonderen, ja einzigartigen Spannungsverhältnis. So sind auf der einen Seite alle Erzählungen über den Holocaust - darüber besteht Konsens in der Forschung - sehr viel stärker als andere geschichtserzählende Werke dem Gebot der historischen Wahrheit verpflichtet (vgl. Kammler) - das gilt auch und in besonderem Maße für die Erzählungen über Janusz Korczak.

Diesem mimetischen Zugang zur Realität, der den Erzählungen auferlegt wird, steht jedoch die Bildebene entgegen. Denn es handelt sich dabei nicht um Fotos, sondern um Zeichnungen, Illustrationen also, die einer sehr „spezifischen graphischen Liminalität“ (Ahrens 49) unterliegen, die ihnen zumindest tendenziell eine Mimesis an Realität verwehrt. Mehr noch: Vor allem auf der Bildebene stellen diese Bilderbücher die genuine Gemachtheit ihrer Darstellungen explizit heraus, d.h. sie sind gerade nicht mit einem Authentizitäts-, sondern vielmehr mit einem Artifizialitätslabel versehen und betonen damit ganz offen ihren fiktionalen Anteil bei der Repräsentation historischer Welten (vgl. Ahrens 49). Damit durchbrechen sie bewusst die Konventionen geschichtlichen Erzählens, denen es ja gerade darum zu tun ist, ihre narrativ-fiktionale Verfasstheit und damit die Tatsache, dass es sich hier um eine symbolische Repräsentation von Geschichte handelt, zu verbergen und widersprechen auf diese Weise zumindest implizit jenen klassischen Aneignungsmodi von Geschichte als Rückholung eines Ereignisses, das im Zuge seiner Narration und Ästhetisierung authentifizierend inszeniert wird (vgl. Ahrens 47-9).

Diesem Authentifizierungsgestus wird jedoch in den Bilderbüchern über Janusz Korczak ebenfalls durchaus Rechnung getragen, etwa durch das Rekurrieren auf und die Abbildung von dokumentarischem Material, wie zum Beispiel durch die Abbildung von Auszügen aus Korczaks Tagebuch 
(Chmielewska N. pag; Jaromir und Cichowska N. pag.), ${ }^{9}$ das immer wieder in die Texte integriert wird, um auf diese Weise gleichsam den Bildern zum Trotz die Authentizität des Erzählten und damit zugleich die Relevanz des zu Erinnernden zu verifizieren. Somit ist den Bilderbüchern über Janusz Korczak nicht nur ein Erinnerungsgebot inhärent, sondern zugleich - wenigstens implizit - zugleich die Aufforderung, auch die mediale Verfasstheit dieser Erinnerungsstrategien in den Blick zu nehmen.

\section{KINDER- UND JUGENDLITERATUR ALS TRANSNATIONALE LITERATUR}

„Lassen Sie uns bei den Kindern anfangen, um diese gänzlich verwirrte Welt langsam wieder ins Lot zu bringen. Die Kinder werden den Erwachsenen den Weg zeigen.“

Das Zitat entstammt dem 1964 erstmals erschienenen Buch Die Kinderbuchbrücke (47) der jüdischen Journalistin und Übersetzerin Jella Lepman (1891-1970). Lepman, eine Kusine von Max Horkheimer, war nach ihrer Emigration bereits 1945 nach Deutschland zurückgekehrt, wo sie 1949 in München die Internationale Jugendbibliothek begründete, die heute eine in ihrer Bedeutung kaum zu überschätzende Sammlung fremdsprachiger Kinder- und Jugendbücher besitzt (Weinkauff und Glasenapp 222). Lepman artikuliert in ihrem Werk einen sehr nachvollziehbaren Wunsch, der die Kinder- und Jugendliteraturszene seit der unmittelbaren Nachkriegszeit bis heute prägt: Durch den internationalen Austausch von Kinder- und Jugendliteratur, so hoffte man, würde sich bei den Leserinnen und Lesern ein positives Bild der Herkunftsländer der Übersetzungen einstellen, so dass die Welt mit dem Heranwachsen neuer Generationen allmählich etwas friedlicher und toleranter würde. Was mit einer Kindheitsutopie seinen Anfang nahm, wird von der Wissenschaft mittlerweile als „Importorientierung der deutschen Kinderund Jugendliteratur" (Weinkauff und Glasenapp 226) gefasst.

Dies wiederum beschreibt ein weiteres Alleinstellungsmerkmal deutschsprachiger Kinder- und Jugendliteratur: In weitaus größerem Umfang als die allgemeine Literatur ist die in deutscher Sprache veröffentlichte Kinder- und Jugendliteratur von nationalen Zugehörigkeiten weitgehend entkoppelt, d.h.

\footnotetext{
${ }^{9}$ Korczaks Tagebuch umfasste nur wenige Monate, von Mai bis August 1942. Sein Sekretär Igor Newerly (1903-1987) rettete es nach Korczaks Deportation vor der Vernichtung und sorgte nach dem Ende des Krieges für dessen Veröffentlichung.
} 
bei knapp 30\% der in Deutschland erscheinenden kinder- und jugendliterarischen Werke handelt es sich um Übersetzungen (Weinkauff und Glasenapp $228 \mathrm{f}$.), die damit eine immense sowohl quantitative wie qualitative Bedeutung innerhalb dieser Literatur besitzen.

Dieser hohe Anteil von Übersetzungen ist auch ein Kennzeichen der kinder- und jugendliterarischen Erzählungen über Janusz Korczak. Sie dienen nämlich keineswegs nur als Zeugnis dessen, was geschehen ist - ungeachtet der Tatsache, dass sie, wie alle Medien ,keine neutralen Träger von ... Informationen" sind (Erll 251) - sie formulieren darüber hinaus, in Text und Bild, sehr explizit einen Erinnerungsanspruch, der allerdings mit einer Neuvermessung allein polnischer oder deutscher Erinnerungsstrategien über die Shoah nicht mehr zu fassen ist. Führt man sich die Gesamtheit der hier in Rede stehenden kinder- und jugendliterarischen Werke über Janusz Korczak noch einmal vor Augen, ${ }^{10}$ so handelt es sich in ihrer überwiegenden Mehrheit um Texte (mit und ohne Bilder), die entweder aus anderen Sprachen ins Deutsche bzw. Polnische übersetzt, oder aus dem Polnischen bzw. Deutschen in andere Sprachen übertragen worden sind - und zu einem kleineren Anteil auch um Erzählungen, die bislang in keine dieser Sprachen übersetzt worden sind (vgl. Adler und Farnsworth, Bernheim, Einat, Kang und Hyeyŏng, Shem-Tov, Spielman und Archambault). Durch diesen Akt der wechselseitigen Übersetzungen werden die Texte zu einem Bestandteil mehrerer Literaturen, darunter auch der polnischen wie der deutschen, oder anders formuliert - bei den Erzählungen über Janusz Korczak handelt es sich, betrachtet man sich die Bandbreite der Übersetzungen, durchaus um Weltliteratur in dem Sinne, dass sie sich nicht nur in ein nationales oder bi-nationales, sondern in ein globales kulturelles Gedächtnis einschreiben.

In diesem Sinne sind die Erzählungen auch zu deuten als eine intertextuell vermittelte, weltweite Kommunikation über Janusz Korczak und die Shoah zwischen Literaturen über Sprach- und Kulturraumgrenzen hinweg, denn im Zuge der Herausbildung einer Weltgesellschaft unterliegen auch die Erinnerungskulturen und Erinnerungsstrategien mehr und mehr transnationalen, ja globalen Einflüssen, die weit über die polnischen und deutschen Grenzen hinausreichen.

Das gilt in besonderem Maße für die Erzählungen über die Shoah - auch und gerade für die kinder- und jugendliterarischen Ausprägungen dieses Erinnerungsmediums. Gerade in diesem Feld war der Anteil an Übersetzungen

\footnotetext{
${ }^{10}$ Vgl. dazu die Bibliographie am Ende des Beitrages mit Angabe ihrer jeweiligen Originalsprache sowie der bislang erschienenen Übersetzungen.
} 
von Beginn an besonders hoch, so dass die Vorstellung von einer ausschließlich aus dem eigenen, nationalen Kulturbestand schöpfenden und sich beständig erneuernden literarischen Erinnerungsliteratur mittlerweile als unhaltbar bezeichnet werden muss. Innovativ an den Erzählungen über Janusz Korczak ist also nicht nur ihr nationale Grenzen überschreitender Charakter, denn hier greifen sie auf bereits bestehende kinder- wie jugendliterarische Traditionen zurück.

Durchaus innovativ gestaltet sich jedoch die Verknüpfung zwischen den Wechselwirkungen der verschiedenen Literaturen und der Fokussierung auf eine historische Figur, die zugleich als Protagonist einer der zentralen Meistererzählungen der Shoah fungiert, oder anders formuliert: Als singulär muss sowohl die Vielzahl, die große sprachliche Bandbreite der Übersetzungen und damit auch die globale Verbreitung der Werke bezeichnet werden als auch die Tatsache, dass diese Übersetzungen mehrheitlich innerhalb eines sehr kurzen Zeitraums getätigt wurden. Als Beispiel sei hier der 2014 in Deutschland erschienene Roman 28 Tage lang von David Safier angeführt, der in weniger als zwei Jahren in insgesamt neun Sprachen übersetzt wurde, darunter auch ins Chinesische ${ }^{11}$.

Nicht zuletzt aus diesen Gründen kann von genuin deutschen oder auch nur deutschsprachigen Erinnerungsstrategien innerhalb der Kinder- und Jugendliteratur nicht mehr gesprochen werden. Auch die in Deutschland veröffentlichten Erzählungen über Janusz Korczak müssen vielmehr als Teil einer Erinnerungskultur betrachtet werden, die zunehmend transnationale, globale Züge trägt. Die globalen Dimensionen, die die (nicht nur) literarische Auseinandersetzung mit der Figur Janusz Korczak in den letzten Jahrzehnten angenommen hat (auch sein wissenschaftliches, publizistisches und literarisches Werk ist mittlerweile in zahlreiche Sprachen übersetzt worden), ist ohne Zweifel zum einen der Tatsache geschuldet, dass es sich um eine historische Persönlichkeit handelt, dessen Leben, Wirken und Handeln durch historische Zeugnisse überliefert und belegt sind. Die ebenso zahlreichen wie vielfältigen Nacherzählungen vor allem des Sterbens von Janusz Korczak, d.h. seiner Entscheidung, die ihm anvertrauten Kinder im Moment der Deportation nicht zu verlassen, denn diese Entscheidung bildet den narrativen Kern aller Erzählungen, machen

${ }^{11}$ Übersetzt wurde der Roman außerdem ins Dänische, Französische, Italienische, Katalanische, Niederländische, Portugiesische, Schwedische und Spanische. Eine Übersetzung ins Amerikanische ist für 2020 angekündigt. Vgl. zu Safier und die Rezeption seines Romans zuletzt Glasenapp und Michaelis-König. 
deutlich, dass diesen Erzählungen nicht nur eine Erinnerungsfunktion zukommt. In ihrer beständigen Wiederholung sind sie zudem dazu angetan, dem (heranwachsenden) Leser Orientierung zu geben, ihm sinnhaftes Verhalten vor Augen zu führen - hinsichtlich eines moralischen Verhaltens, das auch und gerade im Angesicht des Genozids nicht in Frage gestellt werden kann, das klare Grenzen zwischen Gut und Böse sowie eindeutige Wahrheiten offenbart. In der Erinnerung und Beschreibung von Korczaks Handeln gewinnt zugleich das Erzählen über die Shoah eine ihrer zentralen Funktionen zurück, jene der Strukturierung, Ordnung und damit Verfügbarmachung einer für den Einzelnen und damit insbesondere für die Heranwachsenden oftmals nicht mehr erklärbaren Wirklichkeit. Nicht nur auf der Gegenstandsebene, sondern auch in ihrer medialen wie nationalen Vielstimmigkeit verweisen die Erzählungen damit nicht zuletzt explizit auf die zentrale Bedeutung, die der Kinder- und Jugendliteratur mittlerweile weltweit (und damit auch in Polen und Deutschland) als Erinnerungsmedium zugeschrieben wird.

\section{ZITIERTE WERKE}

\section{PRIMÄRLITERATUR}

Adler, David A., and Karen Ritz (Illustr.). Froim: Der Junge aus dem Warschauer Ghetto (1995). Translated from the American by Birgit and Heribert Kupfer, Metropol, 2011.

Adler, David A., and Bill Farnsworth (Illustr.). A Hero and the Holocaust: The Story of Janusz Korczak and His children. Holiday House, 2002.

Appenszlak, Pola. Ha-doktor nish'ar: Roman biografi al Yanush Korczak [Der Doktor bleibt. Biografischer Roman über Janusz Korczak]. Translated from the Polish Manuscript by Chaim Shalom Ben-Awram. Kiryat sefer, 1946 (translated into Yiddish and Argentiniean Spanish).

Bernheim, Mark. Father of the Orphans: The Story of Janusz Korczak. Dutton, 1989.

Bogacki, Tomek. Janusz Korczak: Ein Held der Kinder. 2009. Translated from the American by Egbert Baqué, Knesebeck, 2010 (translated into Hebrew, Japanese, Russian, Spanish).

Bruchfeld, Stéphane, and Paul A. Levine. Erzählt es euren Kindern: Der Holocaust in Europa. 1998. Translated from the Swedish by Robert Bohn and Uwe Danker, C. Bertelsmann, 2000 (translated into Danish, English, Japanese, Turkish).

Chmielewska, Iwona. Blumkas Tagebuch: Vom Leben in Janusz Korczaks Waisenhaus. 2011. Translated from the Polish by Adam Jaromir, Gimpel, 2013 (translated into French, Hebrew, Japanese, Spanish).

Cohen-Janca, Irène, and Maurizio A.C. Quarello (Illustr.). Die letzte Reise: Doktor Korczak und seine Kinder. Translated from the French by Edmund Jacoby, Jacoby \& Stuart, 2015 (translated into American, Czech, Italian, Slovenian, Spanish). 
Du Bouchet, Paule. Sing, Luna, sing: Ein Mädchen erlebt das Warschauer Ghetto. 2004. Translated from the French by Corinna Tramm, Urachhaus, 2010 (translated into Turkish).

Einat, Amela, and Noa Kelner (Illustr.). Stefa Ahuvati: O mifgah echad ve-shalosh preidot [Stefa, meine Liebe. Eine Begegnung und drei Lebwohl]. Glilit, 2014 (translated into Polish).

Gleitzman, Morris. Einmal. 2005. Translated from the Australian by Uwe-Michael Gutzschhahn, Carlsen, 2009 (translated into Chinese, French, Italian, Japanese, Slovenian, Spanish, Hungarian).

Greinecker-Morocutti, Hannelore. "Der Himmel zwischen den Mauern." Chilufim. Zeitschrift für jüdische Kulturgeschichte, vol. 10, 2011, pp. 107-141.

Jaromir, Adam, and Gabriela Cichowska (Illustr.). Fräulein Esthers letzte Vorstellung: Eine Geschichte aus dem Warschauer Ghetto. Gimpel, 2013 (translated into French, Polish).

Kang, Mu-Hong, and Chŏi Hye-yŏng (Illustr.). Chŏnsadŭl ŭi haengjin [Marsch der Engel]. Yangch'ŏl puk, 2011 (translated into English).

Kubert, Joe. Yossel, 19. April 1943: Eine Geschichte des Aufstands im Warschauer Ghetto. 2003. Translated from the American by Horus, Egmont, 2005 (translated into Dutch, French, Italian, Polish, Spanish).

Laird, Christa. Im Schatten der Mauer: Ein Roman um Janusz Korczak. 1989. Translated from the English by Ulla Neckenauer, Ueberreuter, 1990 (translated into Dutch, Japanese, Swedish).

Lawton, Clive A. Die Geschichte des Holocaust. 1999. Translated from the English by Mirjam Pressler, Oetinger, 2002 (translated into Danish, Dutch, French).

Lewin, Waldtraut. Der Wind trägt die Worte: Geschichte und Geschichten der Juden. Volumes 1-2. cbj, 2013.

Mingarelli, Hubert. Das geraubte Licht. 1993. Translated from the French by Hans-Georg Noack, Arena, 1998 (translated into Italian).

Neudeck, Rupert, and Lukas Ruegenberg (Illustr.). Janusz Korczak: Der König der Kinder. Butzon \& Bercker, 2000.

Nürnberger, Christian. Mutige Menschen: Widerstand im Dritten Reich. Gabriel, 2009 (translated into Korean).

Pelz, Monika. "Nicht mich will ich retten": Die Lebensgeschichte von Janusz Korczak. Beltz \& Gelberg, 1985 (translated into Italian, Japanese).

Safier, David. 28 Tage lang: Roman. Kindler, 2014 (translated into Catalan, Chinese, Danish, Dutch, English, French, Italian, Portuguese, Swedish, Spanish).

Sax, Aline, and Caryl Strzelecki (Illustr.). De kleuren van het Getto [Die Farben des Ghettos]. De Eenhoorn, 2011 (translated into English, French).

Shem-Tov, Tami. Ani lo ganav [Ich bin kein Dieb]. Zemorah-Bitan, 2012 (translated into Japanese).

Shepard, Jim. Aron und der König der Kinder: Roman. 2015. Translated from the American by Claudia Werner, Beck, 2016 (translated into Dutch, French, Hungarian, Italian, Serbian, Swedish).

Spielman, Gloria, and Matthew Archambault (Illustr.). Janusz Korczak's children. Kar-Ben, 2007.

Szpilman, Władysław. Śmierć Miasta: Pamiętniki 1939-1945, opracował Jerzy Waldorff, Spółdzielnia Wydawnicza Wiedza, 1946.

Szpilman, Władysław. Das wunderbare Überleben: Warschauer Erinnerungen 1939 bis 1945. Translated from the Polish by Karin Wolff, Econ 1998 (translated into Albanian, Catalan, 
Chinese, Croatian, Czech, Danish, Dutch, English, Finnish, French, Hebrew, Hungarian, Icelandic, Italian, Japanese, Korean, Norwegian, Portuguese, Romanian, Russian, Serbian, Singhalese, Slovenian, Spanish, Swedish, Turkish, Vietnamese).

Stoffels, Karlijn. Mojsche und Reisele (1996). Translated from the Dutch by Mirjam Pressler, Beltz \& Gelberg, 1998 (translated into Danish, Japanese, Spanish).

Szczygielski, Marcin. Flügel aus Papier (2013). Translated from the Polish by Thomas Weiler, Fischer Sauerländer, 2015 (translated into Spanish, Ukrainian).

Vandewijer, Ina. Wie ein Stein in mir: Roman (2008). Translated from the Dutch by Rolf Erdorf, Fischer Sauerländer, 2011.

Weaver, Eva. Jakobs Mantel: Roman (2013). Translated from the English by Werner LöcherLawrence, Droemer, 2013 (translated into Chinese, Czech, Dutch, Italian, Norwegian, Polish, Spanish, Swedish).

Welsh, Renate. Besuch aus der Vergangenheit, Nagel \& Kimche, 1999 (translated into Albanian, Spanish).

Żółkiewska, Wanda. Eine Chance für Heniek. 1978. Translated from the Polish by Kurt Kelm, Der Kinderbuchverlag, 1981 .

\section{SEKUNDÄRLITERATUR}

Ahrens, Jörg. "Neun Thesen zur Möglichkeit einer Rekonfiguration von Geschichte im Comic." Geschichte im Comic. Befunde - Theorien - Erzählweisen, edited by Bernd DolleWeinkauff, Ch.A. Bachmann, 2017, pp. 45-56.

Baskind, Samantha. "Picturing 'The Holiest Thing': Joe Kubert's Children of the Warsaw Ghetto." Visualizing Jewish Narrative. Jewish Comics and Graphic Novels, edited by Derek Parker Royal, Bloomsbury, 2016, pp. 171-84.

Berenstein, Tatiana, editor. Faschismus - Ghetto - Massenmord: Dokumentation über Ausrottung und Widerstand der Juden in Polen während des zweiten [sic!] Weltkrieges. Rütten \& Loening, 1960.

Brüggemann, Theodor, and Hans-Heino Ewers. Handbuch zur Kinder- und Jugendliteratur: Von 1750-1800. Metzler, 1982, pp. 951-1216.

Chłosta-Sikorska, Agnieszka, Hanna Stępniewska-Gębik, and Piotr Trojański, editors. Korczakian Reminiscences: Selected Aspects of the Times \& Legacy of Janusz Korczak. Wydawnictwo Naukowe Uniwersytetu Pedagogicznego, 2014.

Dolle-Weinkauff, Bernd, editor. Geschichte im Comic. Befunde - Theorien - Erzählweisen. Ch. A. Bachmann 2017.

Eisenbach, Artur, editos. Emanuel Ringelblum: Kronika getta warszawskiego wrzesień 1939 styczeń 1943. Spółdzielnia Wydawnicza Czytelnik, 1983.

Erll, Astrid. Kollektives Gedächtnis und Erinnerungskulturen: Eine Einführung. Metzler, 2005.

Ewers, Hans-Heino. Literatur für Kinder und Jugendliche: Eine Einführung in Grundbegriffe der Kinder- und Jugendliteraturforschung. $2^{\text {nd }}$ ed. UTB, 2012.

Frahm, Ole. "Mickey und der Golem. Reflexionen des Holocaust im Comic." Darstellen, Vermitteln, Aneignen. Gegenwärtige Reflexionen des Holocaust, edited by Bettina Bannasch and Hans-Joachim Hahn. Vandenhoeck \& Ruprecht, 2018, pp. 215-56. 
Glasenapp, Gabriele von. “'Nicht mich will ich retten': Kinder- und jugendliterarische Repräsentationen von Janusz Korczak." Neuvermessung deutschsprachiger Erinnerungsstrategien in der Kinder- und Jugendliteratur nach 1990, edited by Bettina Oeste and Ulrike Preußer, Universitätsverlag Rhein-Ruhr, 2017, pp. 93-117.

Glasenapp, Gabriele von, and Hans-Heino Ewers, eds. Kriegs- und Nachkriegskindheiten: Studien zur literarischen Erinnerungskultur für junge Leser. Peter Lang, 2008. Kinder- und Jugendkultur, -literatur und -medien. Theorie - Geschichte - Didaktik 57.

Glasenapp, Gabriele von, and Andree Michaelis König. "Perspektiven: Holocaustliteratur für Kinder- und Jugendliche nach der Jahrtausendwende." Das Gedächtnis an die Shoah in der polnischen und deutschsprachigen Literatur von Autorinnen und Autoren der zweiten und dritten Post-Shoah-Generation, edited by Irmela von der Lühe and Sławomir Jacek Żurek, Towarzystwo Naukowe Katolickiego Uniwersytetu Lubelskiego Jana Pawła II, 2019, pp. 249-63. Pracownia Literatury Polsko-Żydowskiej KUL.

Glasenapp, Gabriele von, and Gisela Wilkending, editors. Geschichte und Geschichten: Die Kinder- und Jugendliteratur und das kulturelle und politische Gedächtnis. Peter Lang, 2005. Kinder- und Jugendliteratur, -literatur und -medien. Theorie - Geschichte - Didaktik 41.

Gödden, Walter. "Hinweise auf Erwin Sylvanus' Korczak und die Kinder." Jüdische Literatur in Westfalen: Vergangenheit und Gegenwart. Symposion im Museum Bökerhof 27. bis 29. Oktober 2000, edited by Hartmut Steinecke, Aisthesis, 2002, pp. 159-73. Veröffentlichungen der Literaturkommission für Westfalen 4.

Greinecker-Morocutti, Hannelore. "Wie ist die Darstellung des Warschauer Ghettos im Comic möglich? Der Himmel zwischen den Mauern." Der dokumentarische Comic: Reportage und Biografie, edited by Dietrich Grünewald, Ch.A. Bachmann, 2013, pp. 139-52.

Kammler, Clemens. "Wahrheit als Kriterium der Wertung von Kinder- und Jugendliteratur zu Nationalsozialismus und Holocaust." Neuvermessung deutschsprachiger Erinnerungsstrategien in der Kinder- und Jugendliteratur nach 1990, edited by Bettina Oeste and U1rike Preußer, Universitätsverlag Rhein-Ruhr, 2017, pp. 9-19.

Lepman, Jella. Die Kinderbuchbrücke. Arbeitsgemeinschaft von Jugendbuchverlegern, 1991.

Lewin, Aleksander. So war es wirklich: Die letzten Lebensjahre und das Vermächtnis Janusz Korczaks. Gütersloher Verlagshaus, 1998. Janusz Korczak. Sämtliche Werke. Ergänzungsband.

Oeste, Bettina, and Ulrike Preußer, editors. Neuvermessung deutschsprachiger Erinnerungsstrategien in der Kinder- und Jugendliteratur nach 1990. Universitätsverlag Rhein-Ruhr, 2017.

Prager, Brad. "The Holocaust without Ink. Absent Memory and Atrocity in Joe Kubert's Graphic Novel Yossel: April 19, 1943." The Jewish Graphic Novel. Critical Approaches, edited bya Samantha Baskind and Ranen Omer-Sherman, Rutgers UP, 2008, pp. 111-28.

Sakowska, Ruta. Die zweite Etappe ist der Tod: NS-Ausrottungspolitik gegen die polnischen Juden, gesehen mit den Augen der Opfer. Ein historischer Essay und ausgewählte Dokumente aus dem Ringelblum-Archiv 1941-1943. Edition Hentrich, 1993. Publikationen der Gedenkstätte Haus der Wannsee-Konferenz 3.

Scheideler, Britta. "Der Friedenspreis des Deutschen Buchhandels." Der Börsenverein des Deutschen Buchhandels 1825-2000: Ein geschichtlicher Aufriss, edited by Stephan Füssel and Georg Jäger, Buchhändler-Vereinigung, 2000, pp. 309-16. 
Schulze, Günter. Nachwort: König Maciuś der Erste: Roman in zwei Teilen für Leser jeden Alters by Janusz Korczak. Translated by Monika Heinker. Kiepenheuer, 1987, pp. 443-54.

Steffen, Katrin. Jüdische Polonität: Ethnizität und Nation im Spiegel der polnischsprachigen jüdischen Presse 1918-1939. Vandenhoeck \& Ruprecht, 2004. Schriften des SimonDubnow-Instituts 3.

Thiele, Jens. "Von der Schwierigkeit, den Holocaust im Bilderbuch darzustellen: Ein Nachtrag zu Rosa Weiss und erste Anmerkungen zu Judith und Lisa."Antisemitismus und Holocaust: Ihre Darstellung und Verarbeitung in der deutschen Kinder- und Jugendliteratur, edited by Werner Anders. Bibliotheks- und Informationssystem der Universität Oldenburg, 1988, pp. 137-47.

Weinkauff, Gina, and Gabriele von Glasenapp. Kinder- und Jugendliteratur. $3^{\text {rd }}$ ed. UTB, 2018.

Wilke, Jürgen. Grundzüge der Medien- und Kommunikationsgeschichte: Von den Anfängen bis ins 20. Jahrhundert. Böhlau. 2000.

Wilkending, Gisela. "Historische, historisch-biografische und autobiografische Romane und Erzählungen für die Jugend." Handbuch zur Kinder- und Jugendliteratur: Von 1850-1900. edited by Otto Brunken, Bettina Hurrelmann, Maria Michels-Kohlhage, und Gisela Wilkending, Metzler, 2008, pp. 537-616.

Zimmermann, Holger. "Kinder- und Jugendliteratur über die Shoah." Darstellen, Vermitteln, Aneignen. Gegenwärtige Reflexionen des Holocaust, edited by Bettina Bannasch and HansJoachim Hahn, Vandenhoeck \& Ruprecht, 2018, pp. 167-92.

\section{„DOKTOR (PO)ZOSTAJE!”: JANUSZ KORCZAK JAKO POSTAĆ LITERACKA}

\section{Streszczenie}

Artykuł bada, jak reprezentowana jest postać literacka Janusza Korczaka w europejskiej literaturze dla dzieci i młodzieży na przestrzeni ostatnich czterdziestu lat. Równocześnie wskazuje na plurimedialność literatury dla dzieci i młodzieży jako medium pamięci, w którym przywołuje się Szoah zarówno na poziomie tekstu, jak i obrazu. W artykule w sposób szczególny podkreślony został transnarodowy charakter tej literatury, manifestujący się także w licznych thumaczeniach opowieści o Januszu Korczaku. Poprzez tłumaczenia teksty te stają się częścią wielu literatur - można tu zastosować termin „literatury światowej” - wpisujących się nie tylko w narodową czy binarodową, ale także globalną pamięć kulturową.

Słowa kluczowe: Janusz Korczak; literatura dla dzieci i młodzieży; Szoah; pamięć kulturowa.

\section{“THE DOCTOR STAYS!”: JANUSZ KORCZAK \\ AS A LITERARY FIGURE}

\section{S u m m a r y}

This paper analyses the literary representation of Janusz Korczak in children's literature of the past 40 years. The characteristics of children's literature as a truly multimedia means of remembrance play a role when both words and pictures commemorate the Shoah. The many stories 
about Janusz Korczak can also point us towards the transnational character of children's literature. Through translation, these stories become part of multiple (national) literatures, so that we can actually speak of a world literature that is not only part of a national or bi-national memory, but of a global, cultural one.

Key words: Janusz Korczak; children's and youth literature; the Shoah; memory culture. 